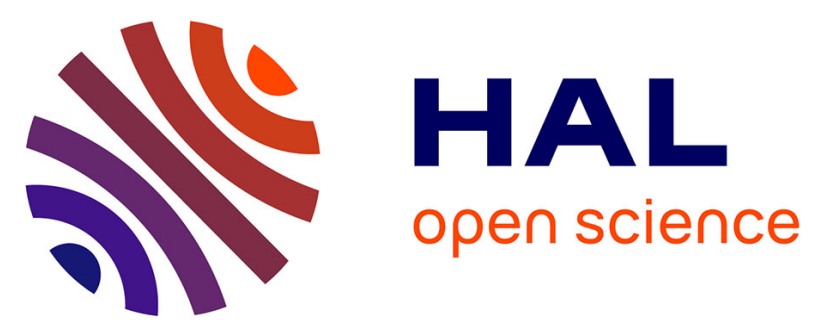

\title{
Numerical Analysis of Thermal Stress in Semi-Transparent Oxide Crystals Grown by Czochralski and EFG Methods
}

Carmen Stelian, Jessica Muzy, Serge Labor, Marc Fivel, Hugues Cabane, Thierry Duffar

\section{To cite this version:}

Carmen Stelian, Jessica Muzy, Serge Labor, Marc Fivel, Hugues Cabane, et al.. Numerical Analysis of Thermal Stress in Semi-Transparent Oxide Crystals Grown by Czochralski and EFG Methods. Crystal Research and Technology, 2018, 54 (1), pp.1800219. 10.1002/crat.201800219 . hal-01953627

\section{HAL Id: hal-01953627 \\ https://hal.science/hal-01953627}

Submitted on 22 Dec 2020

HAL is a multi-disciplinary open access archive for the deposit and dissemination of scientific research documents, whether they are published or not. The documents may come from teaching and research institutions in France or abroad, or from public or private research centers.
L'archive ouverte pluridisciplinaire HAL, est destinée au dépôt et à la diffusion de documents scientifiques de niveau recherche, publiés ou non, émanant des établissements d'enseignement et de recherche français ou étrangers, des laboratoires publics ou privés. 


\title{
Numerical analysis of thermal stress in semi-transparent oxide crystals grown by Czochralski and EFG methods
}

\author{
Carmen Stelian $^{1 *}$, Jessica Muzy ${ }^{1,2}$, Serge Labor ${ }^{2}$, Marc Fivel $^{1}$, Hugues Cabane ${ }^{3}$, Thierry Duffar ${ }^{1}$
}

Received zzz, revised zzz, accepted zzz, Published online zzz

Keywords Czochralski, single crystal growth, stresses, sapphire, computer simulation

Crystal growth of oxides is generally difficult since large curvatures of the growth interface in these systems generate high thermal stress, dislocations and crystal cracking. Three-dimensional numerical modeling is applied to investigate thermal stress distribution in sapphire and langatate $\mathrm{La}_{3} \mathrm{Ta}_{0.5} \mathrm{Ga}_{5.5} \mathrm{O}_{14}$ (LGT) semi-transparent crystals grown by Czochralski (Cz) and Edge-defined Film-fed Growth (EFG) techniques. The analysis of thermal stress distribution in a sapphire ingot of $5 \mathrm{~cm}$ in diameter grown in a Czochralski furnace shows high von Mises stress distributed on large areas in the internal part of the crystal. The distribution of thermal stress is almost axisymmetric, which means that the effect of the anisotropic mechanical properties of sapphire is low. Thermal stress computations for piezoelectric langatate crystals of 5 $\mathrm{cm}$ in diameter grown in a Czochralski configuration show non-symmetrical von Mises distribution with higher stress on one side of the ingot. These numerical results are in agreement with experimental results showing non-symmetrical cracking at the outer surface of the crystal. 3D modeling is applied to investigate thermal stress in a multi-die EFG growth system of white sapphire ribbons. Computations show that the von Mises stress is almost constant with increasing the number of ribbons from 2 to 10 . Two models are applied to simulate the internal radiative heat transfer in the sapphire crystals: P1 approximation and the Rosseland radiation model. Numerical results show that applying Rosseland formula introduces significant errors in temperature field calculations especially in the case of the EFG configuration.

\section{Introduction}

Oxide crystals such as sapphire or langatate $\left(\mathrm{La}_{3} \mathrm{Ta}_{0.5} \mathrm{Ga}_{5.5} \mathrm{O}_{14}\right)$ are used as raw materials for acustooptic-electronic devices. These oxides are usually grown by the Czochralski (Cz), Edge-defined Film-fed Growth (EFG), Heat Exchanger Method (HEM) or Kyropoulos techniques. Crystal cracking and high dislocation density are often reported in $\mathrm{Cz}$ growth of oxides [1-5]. Numerical investigations of thermal stresses in $\mathrm{Cz}$ growth of oxide crystals have shown that crystal cracking and dislocations are related to the temperature field in the furnace and the shape of the crystal-melt interface [6-8]. Oxide crystals are semi-transparent to the infrared radiation by absorbing and emitting the radiative heat. Therefore, the temperature field in the crystal and the shape of the growth interface are significantly affected by the internal radiative heat transfer. Deep convex shapes of the crystal-melt interface are experimentally and numerically reported in these systems [9-12]. Crystal rotation is usually applied to flatten the growth interface and to reduce thermal stresses [1114]. Recently, Stelian et al. [11,12] revealed by means of numerical modeling that applying crystal/crucible rotation in some systems results in distorted shapes of the growth interface and increased stresses in the ingots.

The effect of anisotropic and temperature dependent mechanical properties on thermal stresses distribution in oxide crystals was investigated in $[10,12]$. It was found that the anisotropic effect is significant in $\mathrm{LiNbO}_{3}$ crystals [10] but less important in the sapphire crystals [12].

The objective of the present work is to compare the effect of anisotropic mechanical properties on thermal

\footnotetext{
* Corresponding author: e-mail: steliancarmen@yahoo.com, Phone: +33 476825 213, Fax: ++33 476825249

1Université Grenoble Alpes, CNRS, Grenoble INP, SIMAP, F-38000, Saint Martin d'Hères, France

${ }^{2}$ Le Rubis SA, BP 16, 38560 Jarrie, Grenoble, France

${ }^{3}$ Cristallnnov, Cleanspace 354 Voie Magellan, Sainte-Hélène du Lac, 73800, France
} 
stresses distribution in two oxide crystals (sapphire and LGT) grown by the Czochralski method. Thermal stresses will be also investigated in the case of sapphire ribbons grown in a multi-die EFG system. Finally, the application of two models (P1 approximation and Rosseland radiation model) to simulate the internal radiative heat transfer in sapphire crystals will be discussed.

\section{Model description}

The coupled equations describing the heat transfer, momentum and thermal stresses are solved by using the finite element software COMSOL Multiphysics:

$\rho c_{P}\left(\frac{\partial T}{\partial t}+\vec{u} \cdot \nabla T\right)=k \nabla^{2} T+Q_{R}$

$\rho\left[\frac{\partial \vec{u}}{\partial t}+(\vec{u} \cdot \nabla) \vec{u}\right]=-\nabla p+\mu \nabla^{2} \vec{u}-\rho \vec{g}\left[\beta_{T}\left(T-T_{r e f}\right)\right](2)$

$\sigma_{i j}=C_{i j k l}\left[\varepsilon_{k l}-\alpha_{k l}\left(T-T_{r e f}\right]\right.$

In the above equations, $\rho, c_{p}, k, Q_{R}, p, \mu, \beta_{T}, T, \vec{u}$, $\vec{g}, \sigma_{i j}, \varepsilon_{k l}, C_{i j k l}, \alpha_{k l}$ and $T_{r e f}$ denotes the density, specific heat, thermal conductivity, radiative heat source, pressure, dynamic viscosity, thermal expansion coefficient, temperature, flow velocity, gravity vector, stress tensor components, strain tensor components, components of the elasticity tensor, components of thermal expansion tensor and the reference temperature.

In the case of Czochralski method, temperatures carried out from 2D-axisymmetric global modeling of the furnace are used as boundary conditions for three-dimensional (3D) local modeling of the stress distribution in the crystal. The EFG multi-die system has two orthogonal symmetry planes, so, in this case, $3 \mathrm{D}$ global modeling is applied to compute the temperature field in one fourth of the furnace. Then, 3D local modeling is applied to compute the stress distribution in the sapphire ribbons.

A structured/unstructured combined scheme is applied in 2D global modeling of the furnace. The triangular mesh consists of maximum 15000 domain elements. The mesh used in 3D modeling is unstructured and contains up to 150000 tetrahedral elements. The minimum element size used in 3D thermal stress computations is $0.005 \mathrm{~cm}$.

Temperature computations include heat transfer by conduction, wall-to-wall radiative exchanges and internal radiative heat transfer in the semi-transparent crystals. Two models are applied (and compared) to simulate the internal radiation effect in sapphire crystals grown by $\mathrm{Cz}$ and EFG methods: $\mathrm{P} 1$ approximation and Rosseland radiation model. P1 approximation model considers a radiative heat source $Q_{R}$ in Eq. (1), which is given by:

$$
Q_{R}=a\left(G-4 n^{2} \sigma T^{4}\right)
$$

In the above equation, $a$ is the absorption coefficient, $G$ is the incident radiation, $n$ is the refractive index and $\sigma$ is the Stefan-Boltzmann constant. The melt was considered opaque for both sapphire and LGT computations.

The Rosseland radiation model, which is frequently used in numerical modeling of oxides crystals growth $[15,16]$, assumes an increased thermal conductivity of the semi-transparent crystal:

$k_{\text {eff }}=k_{\text {opaque }}+\frac{16 n^{2} \sigma T^{3}}{3 a}$

$k_{e f f}$ is the effective thermal conductivity of the semitransparent crystal and $k_{\text {opaque }}$ is the thermal conductivity of the opaque crystal.

Heat transfer computations include the latent heat released at the growth interface and the effect of the crystal translation. The shape of the crystal-melt interface is computed by using a deformable mesh technique only in 2D-axisymmetric computations. In the case of 3D modeling of the EFG multi-die system, the shape of the crystal-melt interface was considered flat.

Flow computations include buoyancy convection, Marangoni effect and the forced convection generated by the crystal rotation. The flow is laminar for both $\mathrm{Cz}$ simulated cases. The Grashof numbers $(\mathrm{Gr}=$ $g \beta_{T} \nabla T R^{3} \rho^{2} / \mu$ ) are: $G r=1200$ (for sapphire growth) and $G r=4000$ (for LGT growth).

Thermal stress calculations are three-dimensional and consider the effect of the anisotropic elastic constants. Sapphire is classified as a trigonal class $3 \bar{m}$ crystal with six independent elastic constants: $C_{11}=$ $497 \mathrm{GPa}, C_{12}=162.66 \mathrm{GPa}, C_{13}=117.26 \mathrm{GPa}, C_{14}=$ $22.96 \mathrm{GPa}, C_{33}=501.8 \mathrm{GPa}, C_{44}=147.2 \mathrm{GPa}$ [2]. LGT is a class 32 crystal and has six independent elastic constants: $C_{11}=192 \mathrm{GPa}, C_{12}=111 \mathrm{GPa}, C_{13}=$ $102.9 \mathrm{GPa}, C_{14}=13.8 \mathrm{GPa}, C_{33}=264.1 \mathrm{GPa}, C_{44}=$ $51 \mathrm{GPa}$ [17]. In the present simulations, the z-axis is parallel to the threefold $\mathrm{c}$-axis, and $\mathrm{x}$-axis is parallel to the twofold a-axis of the crystals. The effect of temperature dependent elastic constants and the anisotropy in the thermal expansion coefficient was discussed in a previous work [12]. More details about the numerical and physical model used to simulate crystal growth of oxides are given in $[11,12]$. The physical properties 
of sapphire and LGT are taken from $[2,18]$, and respectively $[17,19]$.

\section{Results and discussion}

\subsection{Crystals grown by the Czochralski tech- nique}

\section{Stress distribution in the sapphire crystals}

The modeling is performed for sapphire crystals of $5 \mathrm{~cm}$ in diameter grown along c-axis in an inductive Czochralski furnace.

Fig.1a shows the temperature and the velocity fields carried out from 2D-axisymmetric simulations performed at a given stage of the growth process (the length of the cylindrical part of the crystal is $L_{c y l}=$ $1.6 \mathrm{~cm}$ ). The shape of the crystal-melt interface was computed by using the deformable mesh technique. Numerical results reveal a deep convex shape of the interface. The computed dimensionless interface deflection $\tau=f / R_{C}=3.6 \mathrm{~cm} / 2.5 \mathrm{~cm}=1.44$ (with $f$ interface deflection and $R_{C}$ - crystal radius) is in agreement with the experimental result $\tau_{\text {exp }}=$ $\mathrm{f} / R_{C}=6 \mathrm{~cm} / 3.8 \mathrm{~cm}=1.57$ of Bruni [3]. The curved shape of the isotherms in the crystal indicates large radial temperature variations that generate high thermal stresses in the sapphire ingot. The flow pattern exhibits one single vortex, with a maximum velocity of $u_{\max }=0.03 \mathrm{~m} / \mathrm{s}$ at the symmetry axis. The isotherms are significantly distorted by the melt flow.

Fig.1b shows the von Mises stress distribution at the outer surface of the crystal. The results are carried out from 3D thermal stress computations that use the temperatures carried out from 2D-axisymmetric global modeling of the furnace. High thermal stresses are observed at the triple crystal-melt-gas line and at the outlet surface of the conical part of the crystal.

Fig. 2 shows the contour lines of von Mises stress distribution plotted in the longitudinal section ( $\mathrm{x}-\mathrm{z}$ plane) and the transversal section ( $\mathrm{x}-\mathrm{y}$ plane at $z=7 \mathrm{~cm}$ ) of the crystal. The contour lines of the von Mises stress are almost axisymmetric in the longitudinal section (Fig. 2a). Large thermal stresses (greater than $200 \mathrm{MPa}$ ) are observed in almost whole crystal volume. The maximum stress value is located at the triple crystal-melt-gas line ( $900 \mathrm{MPa}$ ), while the minimum value $(2.3 \mathrm{MPa})$ is located in the seed. The stress distribution in the transversal section of the crystal reveals a slight asymmetry (Fig. 2b). The difference between the maximum and the minimum values of the von Mises stress at the outer surface of the crystal,
$\Delta \sigma_{\text {Mises }}=\sigma_{\text {Mises }}^{\max }-\sigma_{\text {Mises }}^{\min }=31 \mathrm{MPa}$ represents only $7 \%$ from the maximum stress value. The radial stress distribution along the $\mathrm{x}$-axis in the transversal section of the crystal at $z=7 \mathrm{~cm}$ is plotted in Fig. 2c. High von Mises stress (over $160 \mathrm{MPa}$ ) are distributed in a region of $5 \mathrm{~mm}$ thickness located near the outer surface of the crystal. The critical resolved shear stress varies between $5 \mathrm{MPa}$ and $110 \mathrm{MPa}$ over the temperature range computed in the crystal [20]. That explains high dislocation densities measured in the sapphire crystals grown by the Czochralski technique.

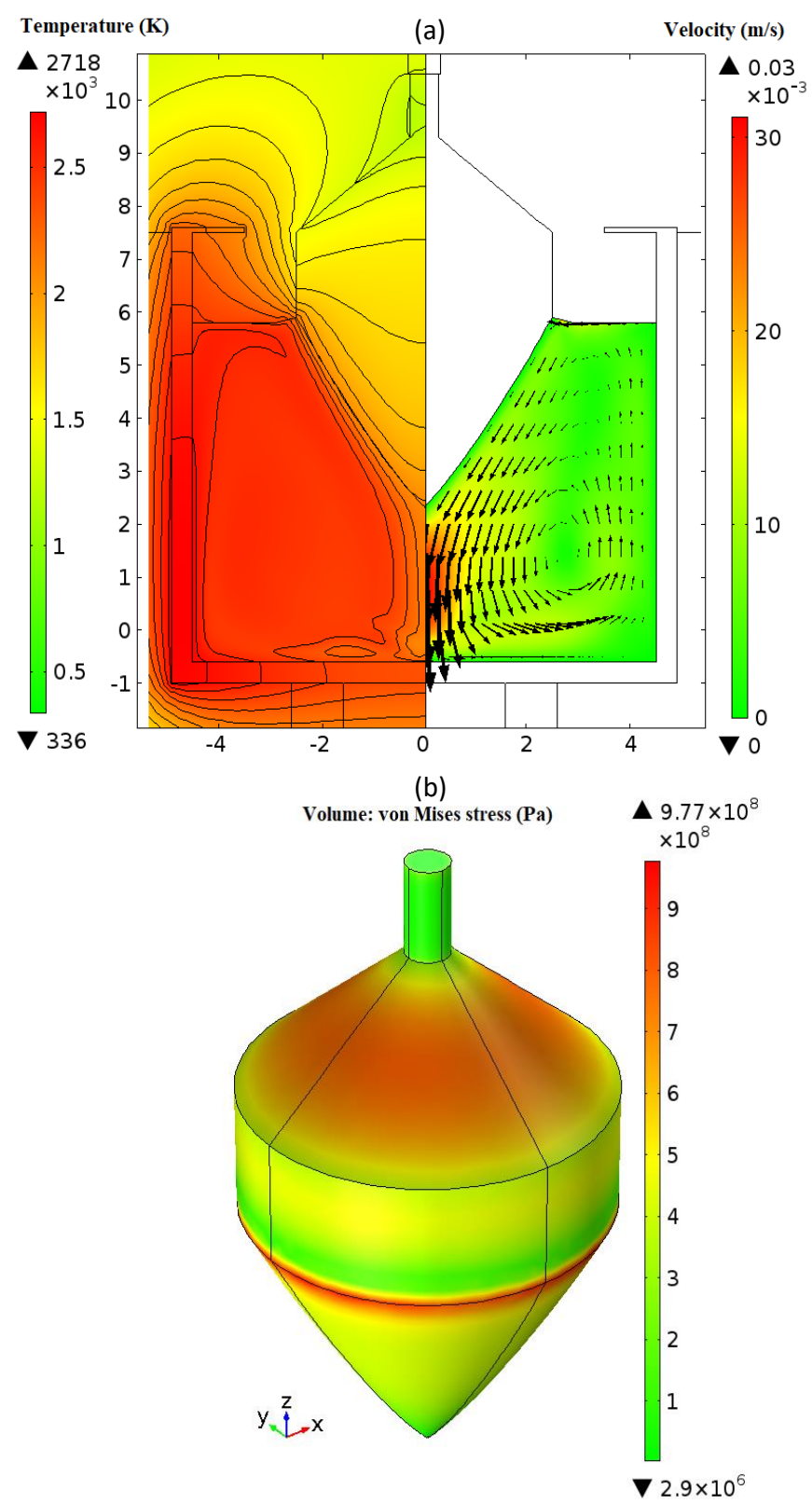

Figure 1 Numerical results obtained in the case of Czochralski sapphire growth: (a) temperature distribution and isotherms (left side), velocity field (right side) carried out from global 2D axisymmetric computations. (b) von Mises stress distribution at the surface of the crystal carried out from local 3D computations. 

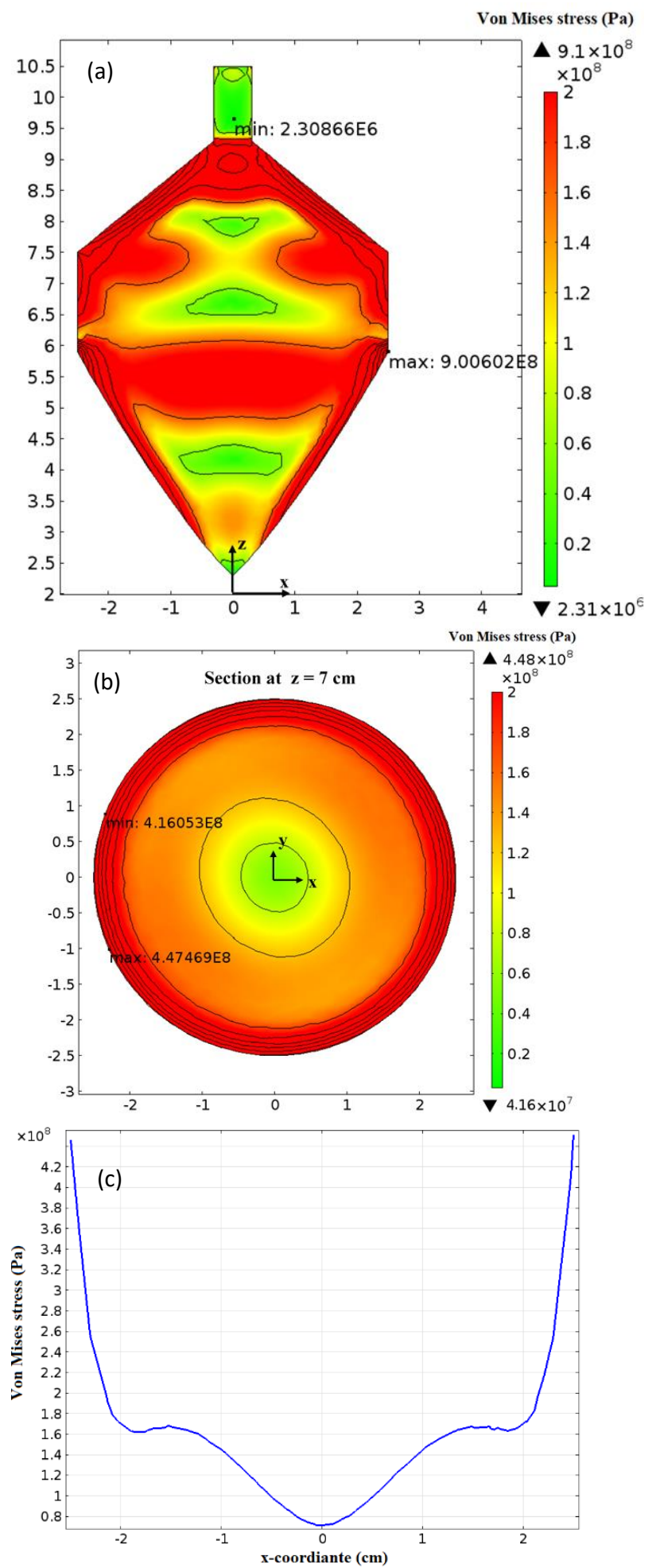

Figure 2 Numerical results obtained in the case of Czochralski sapphire growth: (a) von Mises stress distribution in the longitudinal ( $x$ $z$ ) section of the crystal (maximum and minimum values are shown on the figure); (b) von Mises stress distribution in the transversal ( $x$ y) section of the crystal at $z=7 \mathrm{~cm}$ (maximum and minimum values are shown at the outer surface of the crystal); (c) von Mises stress distribution along the $x$-axis in the transversal $(x-y)$ section of the crystal at $z=7 \mathrm{~cm}$.

\section{Stress distribution in LGT crystals}

Langatate crystals for piezoelectric applications are elaborated in an inductive $\mathrm{Cz}$ furnace. Ingots of $5.4 \mathrm{~cm}$ in diameter are grown along the c-axis from the melt contained in an iridium crucible of $9.2 \mathrm{~cm}$ in diameter. Numerical results carried out from 2D axisymmetric global modeling of the furnace and 3D local modeling of thermal stresses distribution in the LGT ingot are shown in Fig. 3. The crystal-melt interface is less curved in this case: $\tau=$ $f / R_{C}=3 \mathrm{~cm} / 2.7 \mathrm{~cm}=1.11$ (Fig. 3a). The shape of the isotherms in the crystal indicates large radial temperature gradients located near the outer surface of the cylindrical part of the ingot. The recirculating flow is characterized by one single vortex with the liquid moving down through the symmetry axis $\left(u_{\max }=\right.$ $0.03 \mathrm{~m} / \mathrm{s}$ ). Fig. 3 b shows the von Mises stress distribution at the outer surface of the LGT ingot. The maximum value $\sigma_{L G T}^{\max }=511 \mathrm{MPa}$ is smaller as compared to the maximum value computed in the case of sapphire ingot $\sigma_{\text {sapphire }}^{\max }=977 \mathrm{MPa}$. High stress values are observed at the outer surface of the cylindrical part of the ingot.

Fig. 4 shows the von Mises stress distribution in the longitudinal $(x-z$ plane at $y=1 \mathrm{~cm})$ and the transversal ( $x-y$ plane at $z=6.6 \mathrm{~cm}$ ) sections of the crystal. The transversal section $x-y$ at $z=6.6 \mathrm{~cm}$ corresponds to the maximum stress value at the outer surface of the crystal. Numerical results show nonsymmetrical von Mises stress distribution with higher stresses located on one side of the ingot. The difference between the maximum and the minimum stress values at the outer surface of the crystal in the transversal section $\Delta \sigma_{\text {Mises }}=\sigma_{\text {Mises }}^{\max }-\sigma_{\text {Mises }}^{\min }=129 \mathrm{MPa}$ represents $44 \%$ from the maximum stress value (Fig. $4 \mathrm{~b})$. The maximum stress value is reached in a direction parallel to the $y$-axis, while the minimum stress value is located along the $x$-axis. The stress distribution along $\mathrm{x}$-axis in the transversal section at $z=$ $6.6 \mathrm{~cm}$ is plotted in Fig. 4c. It can be observed a nonsymmetrical stress profile and a sharp increase of the von Mises stress (from $40 \mathrm{MPa}$ to $270 \mathrm{MPa}$ ) in a region of $1-2 \mathrm{~mm}$ thickness near the external surface of the crystal. This numerical result could explain the non-symmetrical surface cracking observed in experiments. 


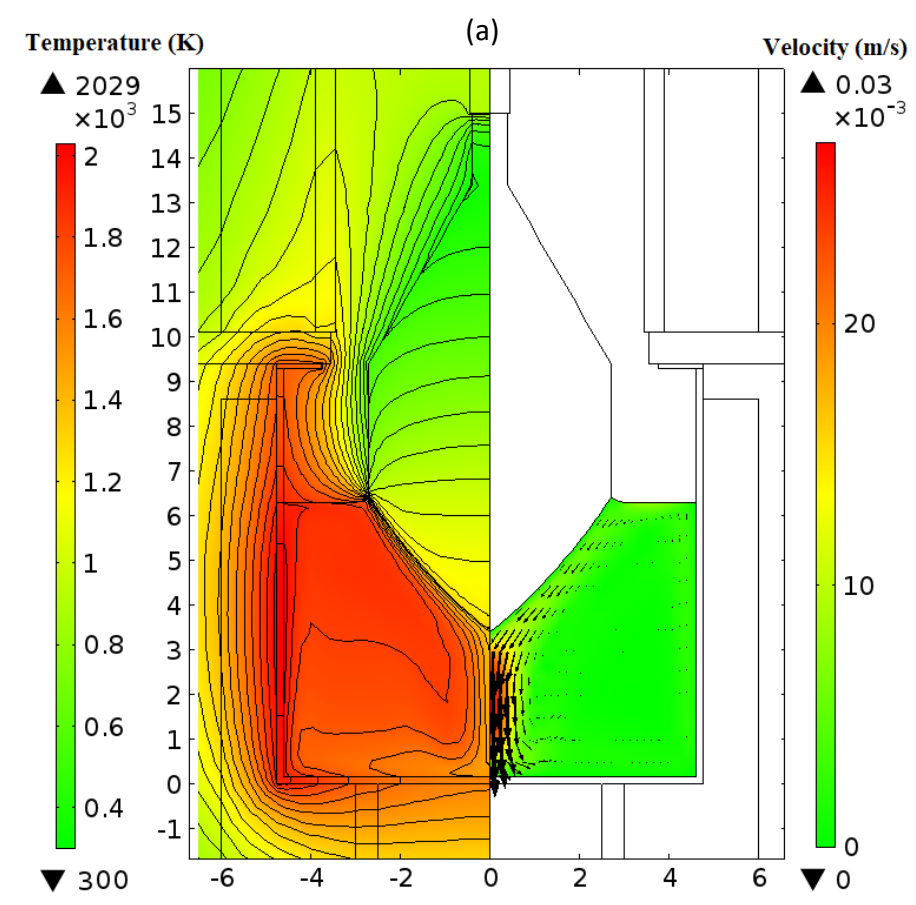

(b)

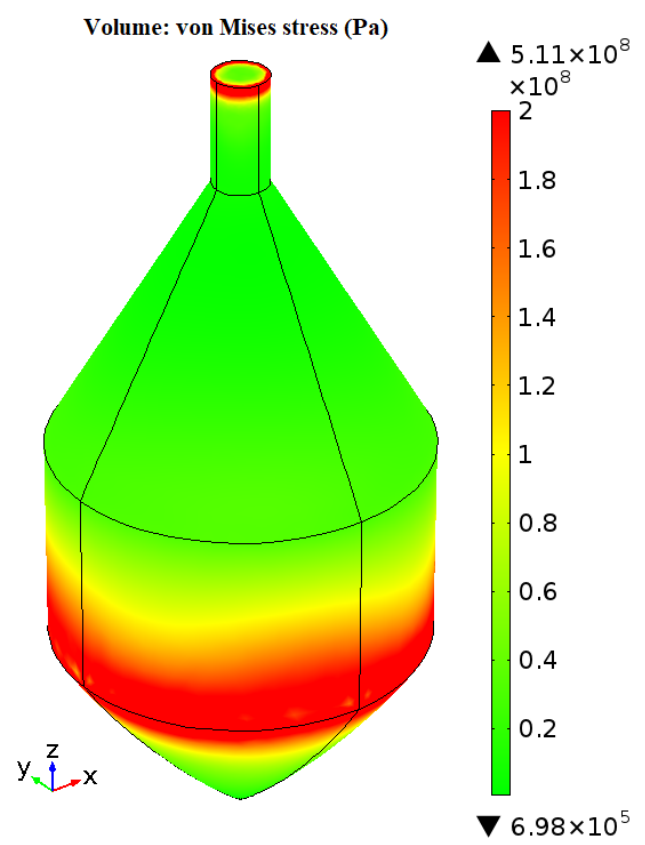

Figure 3 Numerical results obtained in the case of Czochralski LGT growth: (a) temperature distribution and isotherms (left side), velocity field (right side) carried out from global 2D axisymmetric computations. (b) von Mises stress distribution at the surface of the crystal carried out from local 3D computations.
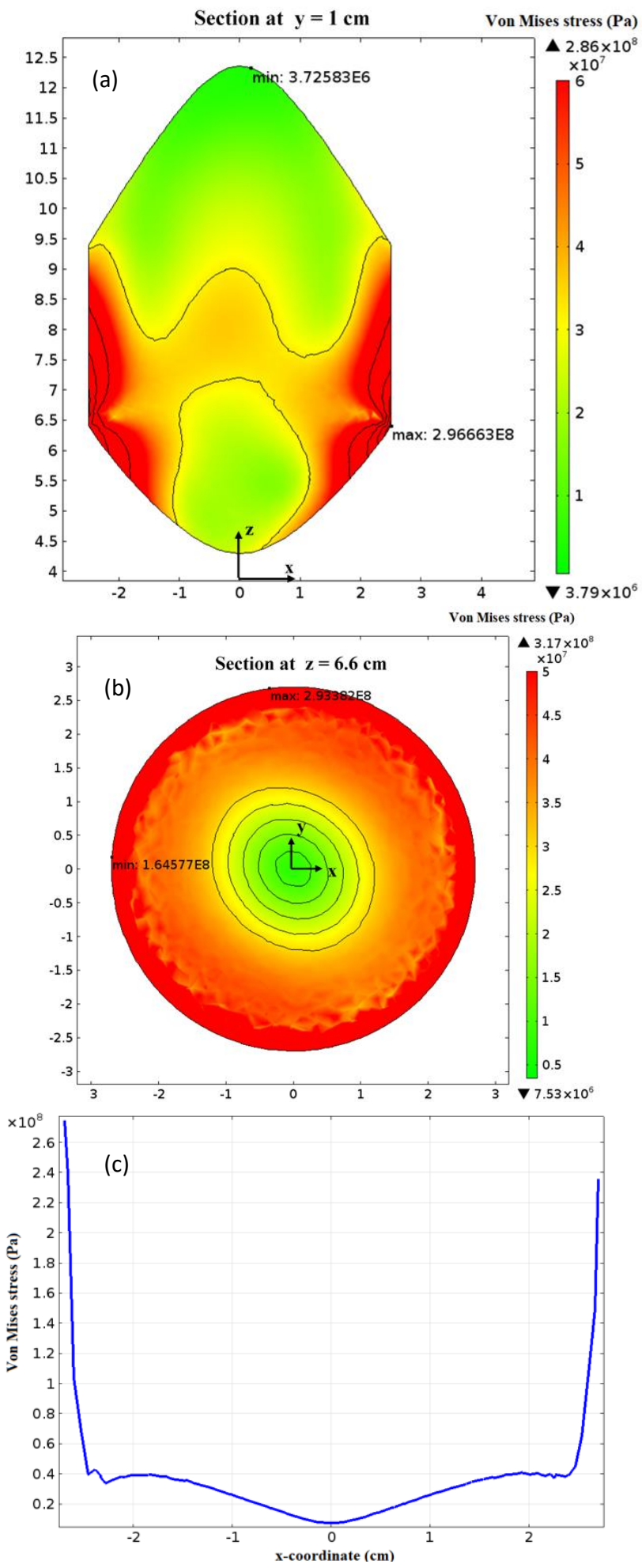

Figure 4 Numerical results obtained in the case of Czochralski LGT growth: (a) von Mises stress distribution in the longitudinal ( $x-z)$ section at $y=1 \mathrm{~cm}$ (maximum and minimum values are shown on the figure); (b) von Mises stress distribution in the transversal ( $x-y)$ section of the crystal at $z=6.6 \mathrm{~cm}$ (maximum and minimum values are shown at the outer surface of the crystal); (c) von Mises stress distribution along $x$-axis in the transversal $(x-y)$ section of the crystal at $z=6.6 \mathrm{~cm}$. 
Fig. 5a shows that the maximum stress value is located at the outer surface of the crystal at $\approx$ $0.25 \mathrm{~cm}$ distance above the triple crystal-melt-gas line in the direction parallel to y-axis. The minimum stress value at the external surface of the crystal is observed along the $x$-axis. Fig. $5 \mathrm{~b}$ shows a LGT ingot of $5.4 \mathrm{~cm}$ in diameter, which was decanted from the melt in order to visualize the shape of the growth interface. The crystal exhibits non-symmetrical surface cracking.

\section{Volume: von Mises stress (Pa)}

(a)

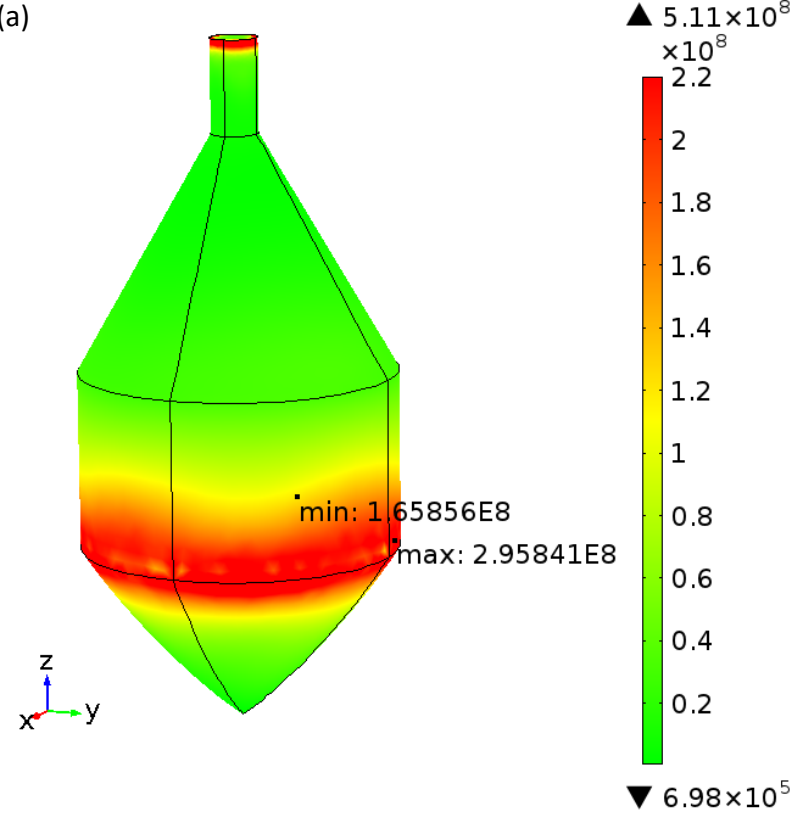

(b)

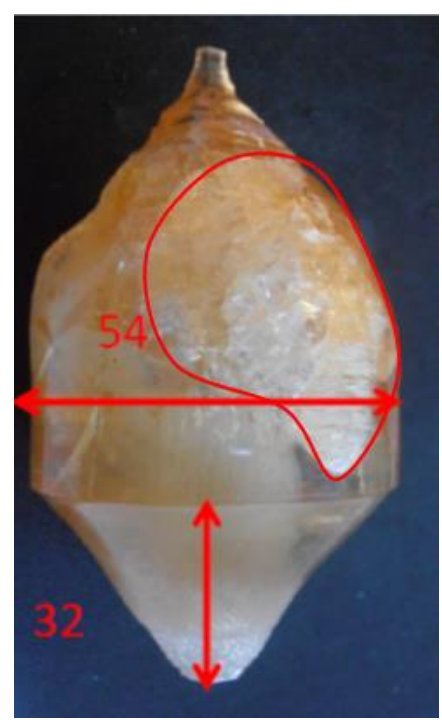

Figure 5 (a) Von Mises stress distribution at the outer surface of the LGT crystal. Maximum and minimum stress values are plotted in a transversal section at $0.25 \mathrm{~cm}$ distance above the crystalmelt-gas line; (b) LGT ingot decanted from the melt which exhibits non-symmetrical surface cracking.

\subsection{Sapphire crystals grown by the EFG tech- nique}

Sapphire ribbons of dimensions $0.8 \mathrm{~cm} \times 4 \mathrm{~cm} \times$ $14.5 \mathrm{~cm}$ are grown in a multi-die EFG system. 3D global modeling is firstly applied to compute the temperature field in the furnace. To minimalize the computational time, the modeling is performed only for a quarter of the real domain by neglecting the convection in the melt. The shape of the crystal-melt interface is not computed in this case, since 3D computations with deformable mesh are too complicated. The meniscus height $h_{m}=0.07 \mathrm{~cm}$ was estimated by applying the analytical formula proposed in [21].

Fig. 6 shows the temperature field carried out from the global modeling of an EFG system with ten molybdenum dies. It can be observed a significant amount of heat lost by the crystals due to the internal radiative effect. The temperatures decrease by $\Delta T=$ $550 \mathrm{~K}$ from the bottom part to the top part of the crystals. Even if the vertical temperature gradients are high in this configuration, the horizontal temperature gradients (which mainly generate the thermal stresses) are minimized due to the heat radiative exchanges between the sapphire ribbons, that homogenize the temperatures in the horizontal direction.

Temperatures computed for EFG systems with 2, 4, 6, 8 and 10 dies are used in the local simulation of the thermal stresses distribution in the sapphire ribbons. The results plotted in Fig. 7 show that the maximum von Mises stress $(\approx 60 \mathrm{MPa})$ remains almost constant with increasing the number of ribbons from 2 to 10. The von Mises stress is higher in the vicinity of the crystal-melt interface, and slightly increases from the center to the outer surface of the crystal. The present results are comparable to the numerical computations of Théodore et al. [22] showing the same von Mises stress distribution map and a maximum value of $\approx 16 \mathrm{MPa}$ in the case of a smaller sapphire ribbon.

Maximum stress values computed in the EFG multi-die system $(\approx 60 \mathrm{MPa})$ are smaller compared to results obtained in the case of $\mathrm{Cz}$ sapphire ingot, which revealed high thermal stresses (over $200 \mathrm{MPa}$ ) spread in almost whole volume of the crystal. 


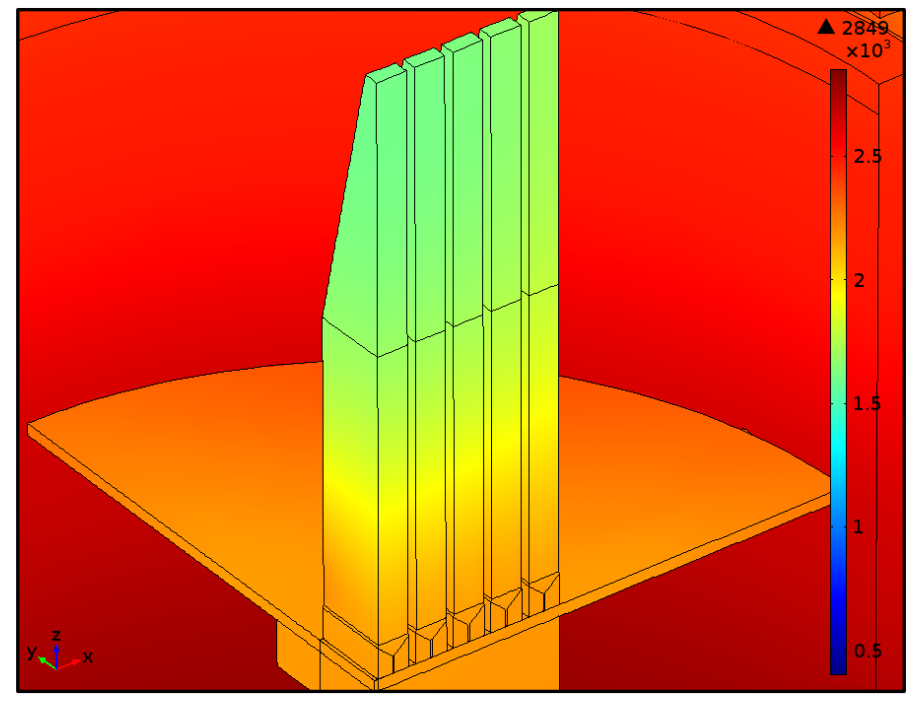

Figure 6 Temperature field carried out from the global modeling of an EFG system with 10 molybdenum dies. Only a quarter of the real domain was simulated.
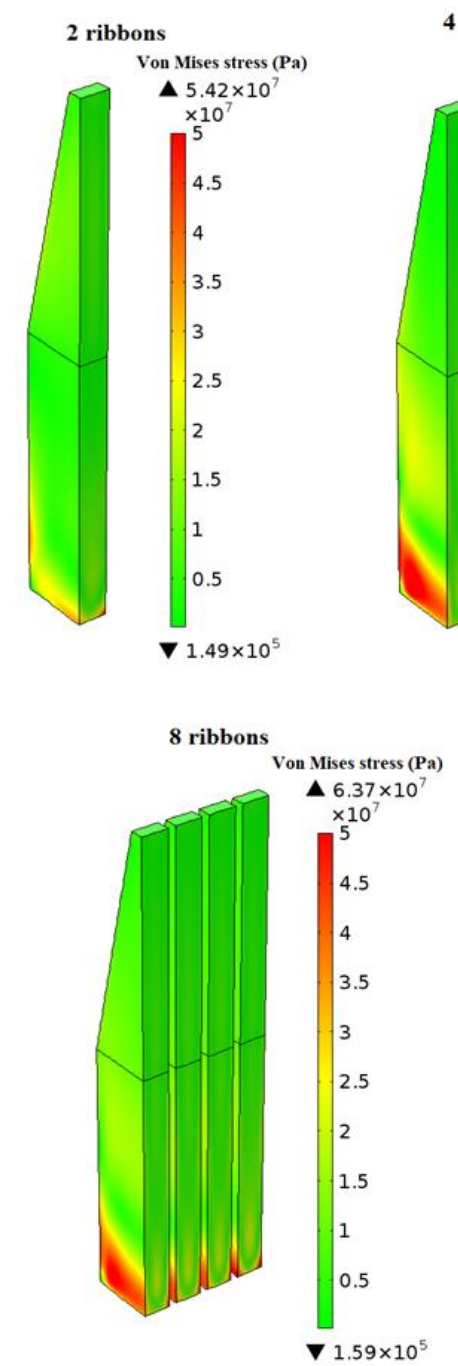

\section{4 ribbons}

Von Mises stress $(P a)$ $\Delta 6.41 \times 10^{7}$

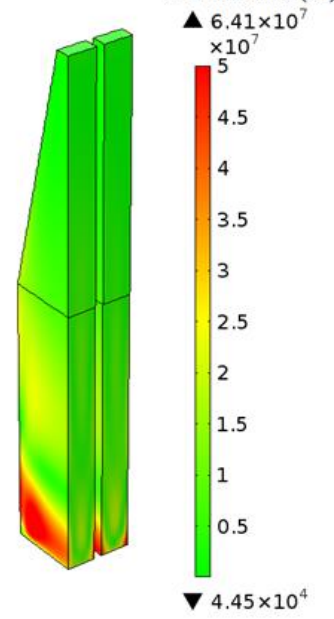

$\nabla .45 \times 10^{4}$

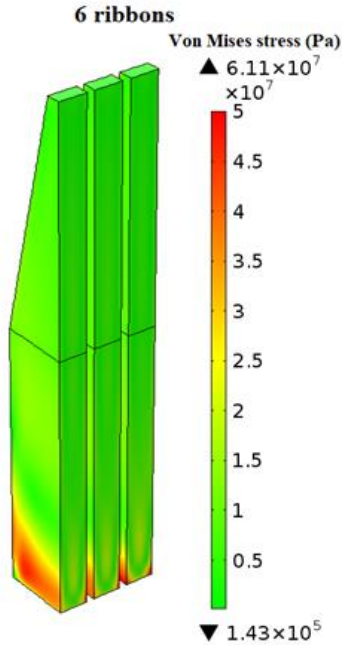

$\boldsymbol{\nabla} 1.43 \times 10^{5}$

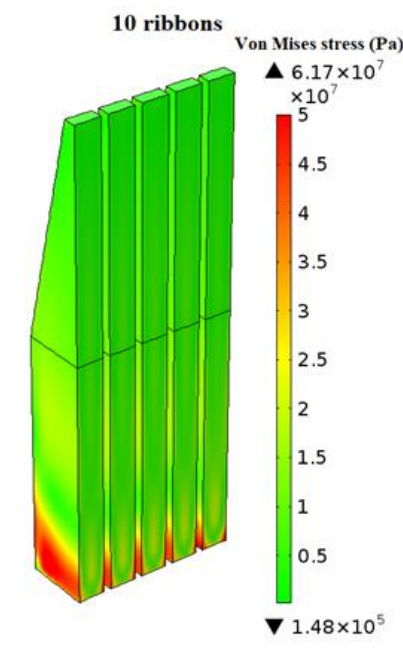

Figure 7 Von Mises stress distribution in sapphire ribbons grown in an EFG multi-die system with 2, 4, 6, 8 and 10 dies. Red color indicates $50 \mathrm{MPa}$. 


\subsection{Application of P1 approximation and Rosseland model to simulate the inter- nal radiative heat transfer}

Two models are usually applied to simulate the internal radiative heat transfer in semi-transparent crystals: P1 approximation and Rosseland radiation model. The Rosseland model, which is most frequently used to simulate the internal radiative effect in sapphire crystals, is based on the artificially enhanced thermal conductivity of the crystal, given by Eq. 5. This model was used for numerical modeling of heat transfer in participating media in $[15,16]$ but the results for the curvature of the growth interface show significant deviations from the experimental observations.

The P1 approximation model takes into account an additional heat source term in Eq. 1 that describes the radiative heat transfer for an absorbing, emitting and scattering medium (the scattering effect is not considered in the present simulations). Numerical modeling based on this model has shown that deep convex shapes of the crystal-melt interface are explained by the internal radiative heat exchanges $[9,11$, $23,24]$. Numerical simulations in $[11,23,24]$ have been validated by comparing the computed shape of the growth interface to the experimental results.

In the present section, P1 approximation and the Rosseland model are applied to simulate the heat transfer in $\mathrm{Cz}$ and EFG growth of sapphire crystals. Fig.8 compares the numerical results carried out from $\mathrm{Cz}$ simulations which use the Rosseland model (Fig. $8 \mathrm{a}$ ) and the P1 approximation (Fig. 8b). The effective thermal conductivity of the sapphire crystal $\left(k_{e f f} \approx\right.$ $500 \mathrm{~W} / \mathrm{K} \cdot \mathrm{m}$ ) was estimated by using Eq. 5 (with $a=$ $20 \mathrm{~m}^{-1}$ and $\left.n=1.77\right)$. The shape of the growth interface was computed by using the deformable mesh technique. During a transient time $(50-300 s)$, the mesh is deformed in such way as the surface between the crystal and the melt (shown on the figure at time $=0 \mathrm{~s}$ ) takes the shape of the solidification isotherm. Fig. 8a shows the numerical plot at the beginning ( $t=$ $0 \mathrm{~s}$ ) and the end ( $t=300 \mathrm{~s}$ ) of the transient computation with deformable mesh. The dimensionless interface deflection $\tau=f / R_{C}=2.4 \mathrm{~cm} / 2.5 \mathrm{~cm}=0.96$ is smaller than observed in experiments [3]. Temperatures in the crystal are homogeneous and close to the melting point, which indicate that the amount of heat lost by the crystal is very small.

The results carried out by applying the P1 approximation show much curved interface (Fig. 8b). The dimensionless interface deflection $\tau=$ $f / R_{C}=3.6 \mathrm{~cm} / 2.5 \mathrm{~cm}=1.44$, is much closer to the experimental result of Bruni [3]. The temperatures in the crystal decrease significantly due to the internal radiative effect. That generates large temperature gradients in the crystal, and, consequently, high thermal stresses, dislocations and cracking.

Numerical results obtained by applying the Rosseland and P1 approximation models in the case of EFG growth of 10 sapphire ribbons are plotted in Fig. 9a and, respectively, Fig. 9b. The crystal-melt interface was considered plane in these simulations. The power in the furnace was varied until the melting point is reached in the liquid meniscus corresponding to the die 6 , which is nearest from the symmetry plane (central dies are labeled as 5 and 6). Fig. 9a shows the temperature distribution in the vertical $(\mathrm{x}-\mathrm{z})$ section at the center of the dies (symmetry plane). The red line represents the solidification isotherm. It can be observed that the temperatures decrease significantly in the horizontal direction (from the ribbon 10 to the ribbon $6)$. The solidification isotherm is located in the liquid meniscus only for the dies 6-8, then deviates far from the liquid meniscus for the dies 9 and 10. That should make impossible the simultaneous growth of 10 ribbons, which is in contradiction with our experimental results. Fig. $9 \mathrm{~b}$ shows more realistic results carried out from simulations which use the P1 approximation. In this case, the temperatures decrease on the vertical direction, from the bottom part to the top part of the ribbons. The solidification isotherm is located near the crystal-melt interfaces for all the ribbons.

\section{Conclusions}

Three-dimensional numerical simulations were performed to investigate the effect of the anisotropic elastic constant on thermal stresses distribution in sapphire and piezoelectric langatate crystals grown by the Chochralski and EFG techniques.

The modeling performed in the case of sapphire crystals grown in an inductive $\mathrm{Cz}$ configuration shows large thermal stresses distributed almost symmetrically in the whole volume of the crystal. This result could explain high dislocation densities measured in the sapphire crystals grown by this technique. 
(a)

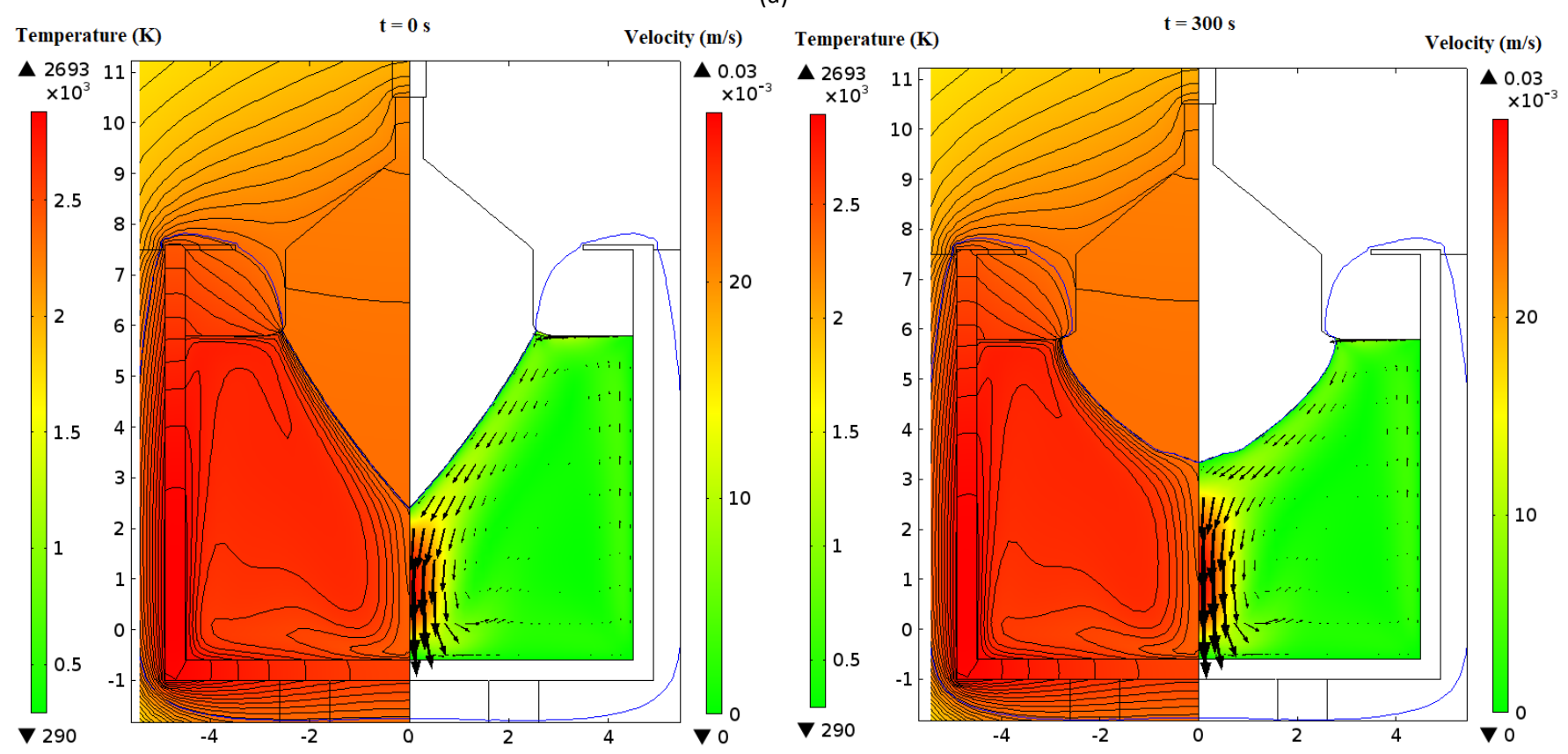

(b)
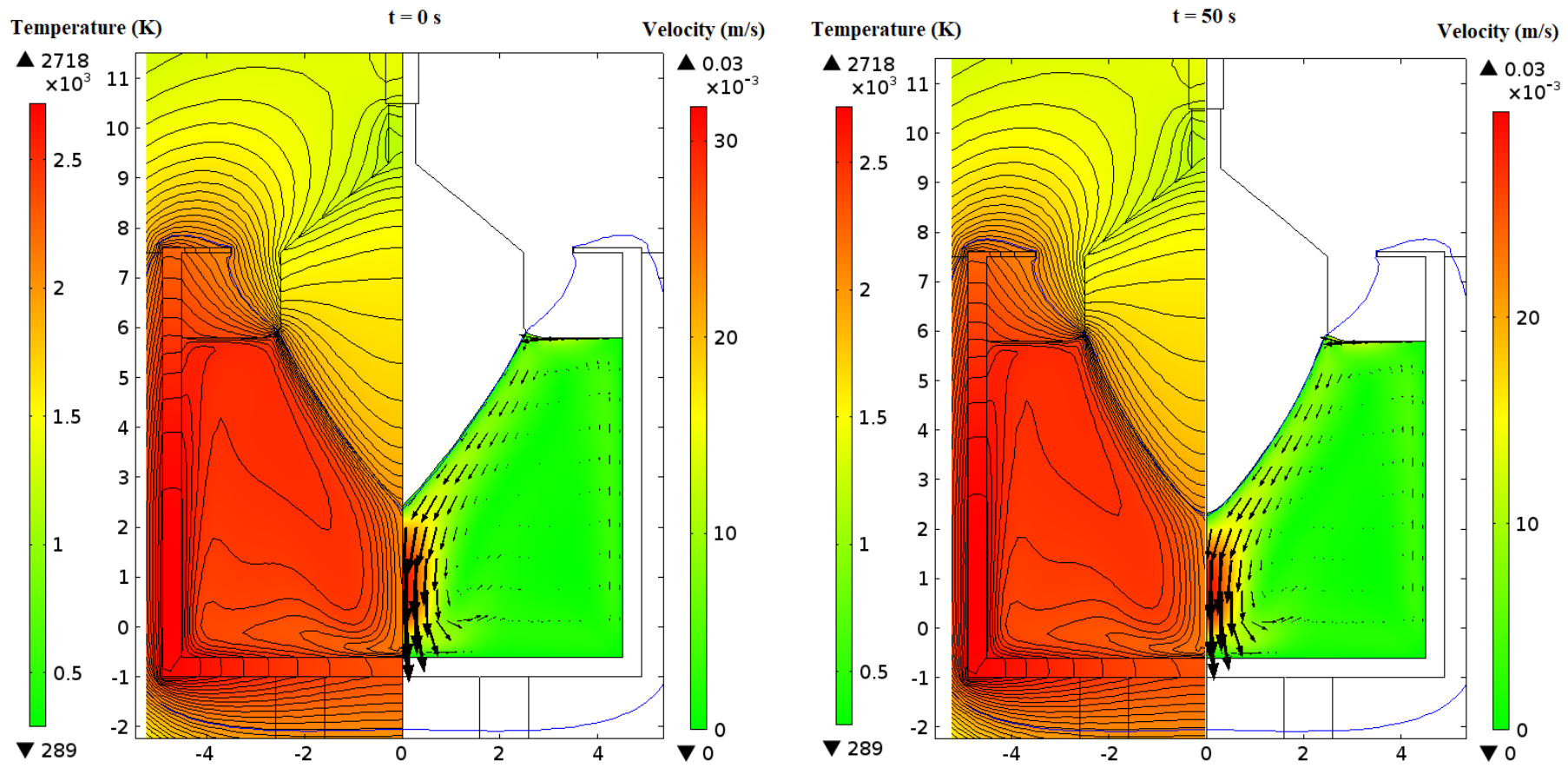

Figure 8 Temperature and velocity fields computed at the beginning ( $t=0 \mathrm{~s})$ and the end $(t=300 \mathrm{~s}, \mathrm{t}=50 \mathrm{~s})$ of transient simulations with deformable mesh carried out for a Czochralski sapphire growth system by applying: (a) Rosseland radiation model; (b) P1 approximation. The blue line represents the solidification isotherm. 
(a)

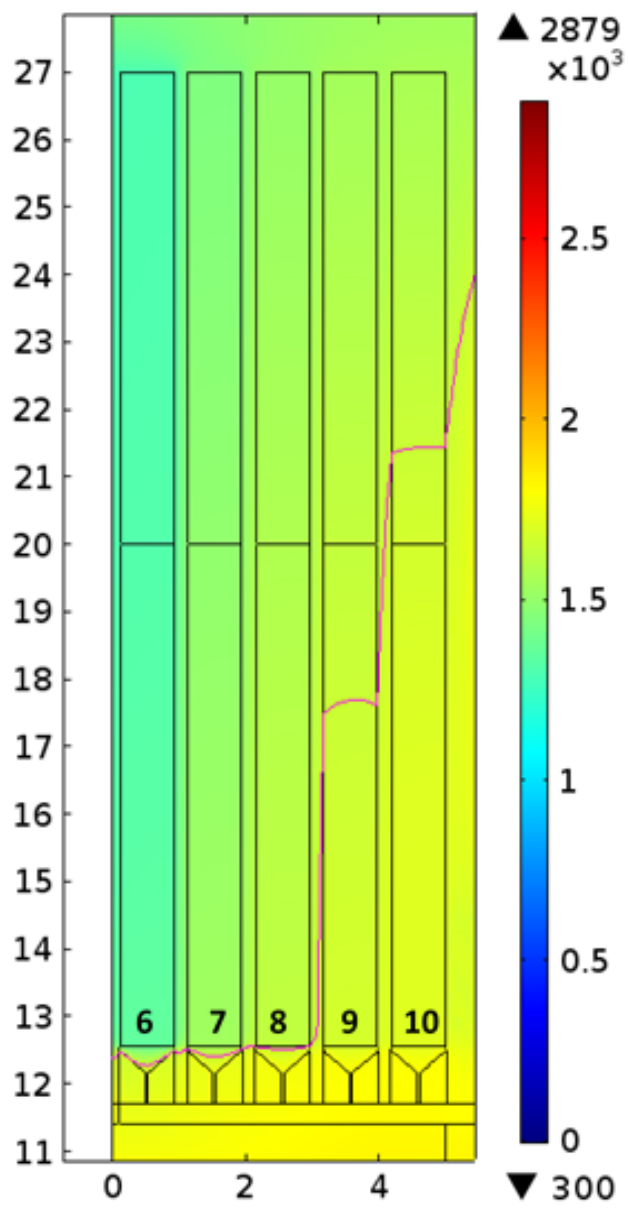

(b)

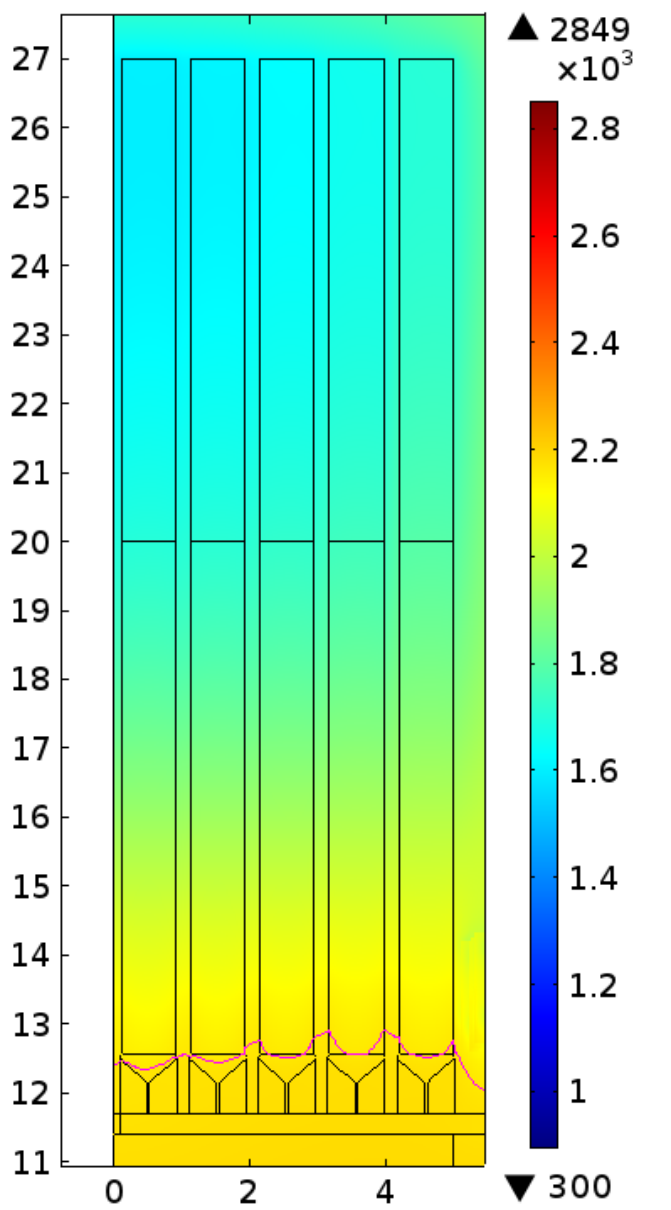

Figure 9 Temperatures computed in the case of EFG growth of 10 sapphire ribbons by applying: (a) Rosseland radiation model; (b) P1 approximation. The red line represents the solidification isotherm.

The computations performed in the case of langatate crystals grown in a $\mathrm{Cz}$ configuration show non-symmetrical stress distribution with maximum stress located at one side of the crystal. The von Mises stresses are relatively small in the internal part of the crystal, but increase significantly in a thin region of 1$2 \mathrm{~mm}$ located near the outer surface of the crystal. This explains non-symmetrical cracks experimentally observed at the external surface of the crystal. Large temperature gradients at the outer surface of the crystal could be generated by the additional thermal shields inserted around the crystal in this configuration.
Thermal stress computations performed in the case of a multi-die EFG system show that the distribution and the maximum von Mises values are almost unchanged with increasing number of ribbons from 2 to 10 . This is explained by the radiative heat exchanges between the ribbons that homogenize the temperatures in the horizontal direction. The maximum stress values are smaller as compared to the case of $\mathrm{Cz}$ sapphire growth, which predicts lower dislocation densities in sapphire ribbons grown by the EFG technique.

The application of two models (P1 approximation and Rosseland model) to simulate the internal radiative heat transfer was investigated in the case of sapphire crystals grown by $\mathrm{Cz}$ and EFG techniques. 
The present results show that the Rosseland model introduces significant errors in heat transfer computations, especially in the case of the EFG system. It is found that the interface curvatures are generally underestimated when the Rosseland model is applied to simulate $\mathrm{Cz}$ growth of sapphire. EFG thermal computations using the Rosseland model give unrealistic results for temperatures distribution and the position of the solidification isotherm. On the other hand, the application of P1 approximation model give more realistic results, which are validated by comparison to experiments. It is concluded that the Rosseland model, which is still frequently used in numerical works, is not appropriate to simulate the internal radiative effect in semi-transparent crystals.

\section{References}

[1] C.D. Brandle, J. Cryst. Growth 264, 593 (2004).

[2] T. Vodenitcharova, L.C. Zhang, I. Zarudi, Y. Yin, H. Domyo, T. Ho and M. Sato, J. Mater. Process. Technol. 194, 52 (2007).

[3] F.J. Bruni, Cryst. Res. Technol. 50, 133 (2015).

[4] M.S. Akselrod and F.J. Bruni, J. Cryst. Growth 360, 134 (2012).

[5] J. Luo, D. Shah, C.F. Klemenz, M. Dudley and H. Chen, J. Cryst. Growth 287, 300 (2006).

[6] T. Tsukada, K. Kakinoki, M. Hozawa, N. Imaishi, K. Shimamura and T. Fukuda, J. Cryst. Growth 180, 543 (1997).

[7] S. E. Demina and V.V. Kalaev, J. Cryst. Growth 320, 23 (2011).

[8] M. Kobayashi, T. Tsukada and M. Hozawa, J. Cryst. Growth 241, 241 (2002).

[9] Q. Xiao and J.J. Derby, J. Cryst. Growth 139,147 (1994).

[10] M. Miyazaki, H. Uchida, T. Tsukada and T. Fukuda, J. Cryst. Growth 162, 83 (1996).

[11] C. Stelian and T. Duffar, Cryst. Res. Technol. 52, 1700176 (2017).

[12] C. Stelian, G. Sen and T. Duffar, J. Cryst. Growth 499, 77 (2018).

[13] J. Hur, X.F. Han, D.S. Song, T.H. Kim, N.J. Lee, Y.J. Jeong, and K.W. Yi, J. Cryst. Growth 385, 22 (2014).

[14] T.P. Nguyen, Y.T. Hsieh, J.C. Chen, C. Hu and H.B. Nguyen, J. Cryst. Growth 468, 514 (2017).

[15] C.W. Lu, J.C. Chen, C. H. Chen, C. H. Chen, W. C. Hsu and C.M. Liu, J. Cryst. Growth 312, 1074 (2010).

[16] M.H. Tavakoli and H. Wilke, J. Cryst. Growth 275, e85 (2005).
[17] P.M. Davulis, "Characterization of the Elastic, Piezoelectric, and Dielectric Properties of Langatate At High Temperatures Up To $900{ }^{\circ} \mathrm{C}$ " (2013). Electronic Theses and Dissertations. Paper 2064.

[18] C.H. Chen, J.C. Chen, C.W. Lu and C.M. Liu, J. Cryst. Growth 318, 162 (2011).

[19] O.M. Kugaenko, S.S. Uvarova, S.A. Krylov, B.R. Senatulin, V.S. Petrakov, O.A. Buzanov, V.N. Egorov and S.A. Sakharov, Bull. Russ. Acad. Sci.: Phys. 76, 1258 (2012).

[20] K.P.D. Lagerlof, A.H. Heuer, J. Castaing, J.P. Riviere, and T.E. Mitchell, J. Am. Ceram. Soc. 77, 385 (1994).

[21] L. Carroz and T. Duffar, Cryst. Res. Technol. 50, 473 (2015).

[22] F. Théodore, T. Duffar and F. Louchet, J. Cryst. Growth 198/199, 232 (1999).

[23] C. Stelian, A. Nehari, I. Lasloudji, K. Lebbou, M. Dumortier, H. Cabane and T. Duffar, J. Cryst. Growth 475, 368 (2017).

[24] C. Stelian, M. Velázquez, P. Veber, A. Ahmine, J. B. Sand, G. Buşe, H. Cabane and T. Duffar, J. Cryst. Growth 492, 6 (2018). 
Table of Contents

Modeling of Czochralski growth of sapphire

Page xx-yy (for typesetter)

C. Stelian, J. Muzy, S. Labor, M. Fivel, H. Cabane, and T. Duffar

Numerical analysis of thermal stress in semi-transparent oxide crystals grown by Czochralski and EFG methods

Modeling of Czochralski growth of sapphire
crystals shows large thermal stresses distributed symmetrically in the whole volume of the crystal. That could explain high dislocation densities measured in the sapphire ingots. Computations performed in the case of langatate crystals show non-symmetrical stress distribution, with maximum stress located at one side of the crystal. That explains non-symmetrical surface cracking observed in the experiments. Numerical results show that the Rosseland model is not appropriate to simulate the internal radiative effect in semi-transparent crystals.

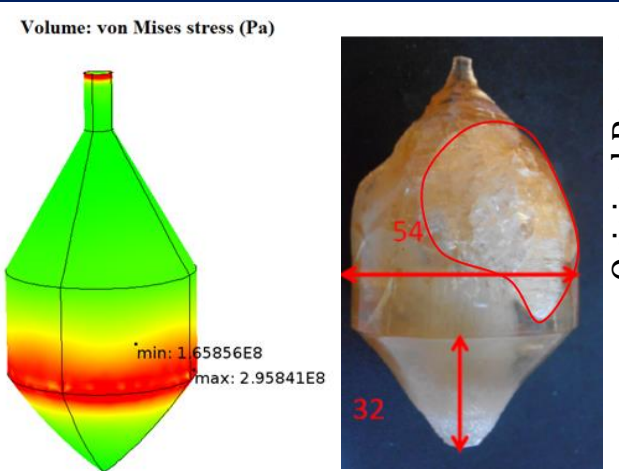

